\title{
CDISC SDTM Implementation Guide Version 3.1.2 Amendment 1
}

National Cancer Institute

\section{Source}

National Cancer Institute. CDISC SDTM Implementation Guide Version 3.1.2 Amendment

1. NCl Thesaurus. Code C161434.

Amendment 1 of the 3.1.2 version of the CDISC Study Data Tabulation Model (SDT M) implementation guide. 